\title{
ANXIETY, BURNOUT AND DEPRESSION LEVELS ACCORDING TO SEX AND YEARS OF WORK EXPERIENCE IN ITALIAN NURSES ENGAGED IN THE CARE OF COVID-19 PATIENTS
}

\begin{tabular}{l} 
Elsa Vitale ${ }^{* 1}$, Salvatrice Casolaro ${ }^{2}$ \\
${ }^{1}$ Registered Nurse at the Department of Mental Health, ASL Bari, Italy. Contract professor for \\
the basic and specialist degree course in nursing at the University of Bari. \\
${ }^{2}$ Healthcare Assistant. Organizational Position of the Metapontine District Area, Policoro \\
Materan Hill, Matera Health Company, Matera, Italy. \\
\hline \hline
\end{tabular}

\begin{abstract}
Aim: To evaluate burnout, anxiety and depression levels in nurses directly involved in the care of Covid-19 patients. Furthermore, particular attention was paid to the existence of any differences between male and female nurses to evaluate whether the variables considered can be influenced by the gender variable.

Design: A cohort case-report study was carry out.

Sample: All Italian nurses who were at front line in the care of the Covid-19 patients were involved.

Measurement: The on line questionnaire collected data as regards: gender and years of work experience; the Maslach Burnout Inventory questionnaire (MBI) and the Hospital Anxiety and Depression Scale (HADS) questionnaire.

Results: Certainly the experience of the pandemic was very emotional for nurses engaged in the front line in the care of Covid-19 patients. However, anxiety and depression levels turned out to be normal, while some nurses experienced them in the dimensions of emotional exhaustion and depersonalization of the burnout rating scale.

Conclusion: The practical implication was the implementation of support policies for nurses in tackling the problem of burnout more than that of anxiety or depression.
\end{abstract}

Keywords: Anxiety level; Burnout level; Covid-19; Depression level; Nurse; Sex Work Experience

\footnotetext{
* Correspondence concerning this article should be addressed to Elsa Vitale, Department of Mental Health, Local Healthcare Company Bari, Italy, Via X marzo, 43, 70026 Modugno, Bari.

Telephone: +3339910154

E-mail: vitaleelsa@libero.it
} 


\section{Introduction}

In December 2019, a highly infectious serious acute respiratory syndrome caused by a novel coronavirus (SARS-CoV-2) emerged in Wuhan, China. On March $11^{\text {th }} 2020$, the World Health Organization (WHO) declared COVID-19 a pandemic (Huang et al., 2020).

Previous studies on the SARS epidemic, in 2003, showed that exposed health workers reported experiencing high levels of stress, anxiety and depression; symptoms, with the risk of long-term psychological implications (Chung et al., 2005; Mok et al., 2005; Suzuki et al., 2006).

As demonstrated in literature, healthcare professionals engaged in the forefront of treating Covid-19 patients every day are exposed to a high risk of infection and long and distressing shifts to meet health needs. In summary, they are exposed to a source of prolonged distress and their individual coping skills may not be enough to cope with it (Bohlken et al., 2020; Chen et al., 2020; Du et al., 2020; Murthy et al., 2020; Tan et al., 2020; Vitale et al., 2020; Wu et al., 2020).

Already in the literature there are numerous studies that report important burnout, anxiety and depression levels in healthcare professionals involved in the care of Covid-19 patients. However, the literature does not report the exact link between these factors (Zhang et al., 2020; Wang et al., 2020; Usher et al, 2020; Vitale et al. 2020). However, even in the absence of particular health emergency situations, nursing work itself is considered alienating. Several studies in literature report in fact high levels of burnout, especially among nurses with a greater number of years of work experience (Mathieu et al., 2014; Van Zyl et al., 2018; Craigie et al., 2016; Vitale et al., 2020). Instead, there are many studies that report high levels of anxiety and depression among nurses during previous health emergency situations, such as SARS (Liu et al., 2012; Cheung et al., 2020) or the spread of the Ebola virus (Lehmann et al., 2015; Kreuels et al., 2014).

The present study aimed to evaluate burnout, anxiety and depression levels in nurses directly involved in the care of Covid-19 patients. Specifically, we investigated levels of burnout, anxiety and depression among Italian front line nurses during the pandemic, also according to sex and years of work experience and finally, relationships between all variables considered in this cohort survey.

\section{Materials}

\section{The questionnaire}

The questionnaire contained a first part of demographic data, as:

- Sex: participants were divided according to females' and males' subjects;

- Years of work experience, divided into three groups, namely: from 0 to 10 years, from 11 to 25 years and above 25 years. 
Then, the second part of the questionnaire included the Maslach Burnout Inventory (MBI) questionnaire (Schaufeli et al., 2001), which was administered to evaluate the burnout level among nurses who were in direct contact with Covid-19 patients. It described burnout in a three-dimensional syndrome, as:

- Emotional Exhaustion (EE), which included items numbers: 1,2,3,6,8,13, 14,16,20. This condition consisted in felling physical overexertion and emotional fatigue as a result of the continuous interactions required between the worker and the users of the service;

- Depersonalization (D) or the presence of cynical attitudes and responses towards the people to whom the services were provided, which included items number: $5,10,11,15,22$;

- Personal Achievement (PA) or the lack of confidence and negative selfesteem resulting from meetings with unrewarding situations, which included items number: 4, 7, 9, 12, 17, 18, 19, 21.

Participants were asked to rate from 0 (never) to 6 (always) the frequency in which they experienced feelings described in each of the 22 -items. For each subdimension a risk category was assessed, as low, medium and high category risk. Specifically, for EE sub-dimension, values below 17 identified a low category risk; values included from 18 to 29 designed a medium risk and values above 30 classified a high risk. As regards D scores, values below 5 identified a low category risk, for values included from 6to 11 designed a medium category risk and values above 12 classified a high risk. Finally, as concern PA sub-dimension, values above 40 identified low category risk, values included from 34 to 39 classified a moderatemedium risk and values below 33 indicated high category risk.

The third and final part of the questionnaire included the assessment of states of anxiety and depression by administering the Hospital Anxiety and Depression Scale (HADS, Djukanovic et al., 2017). Nurses were asked to indicate how they have been feeling during the Covid-19 pandemic period among the different options presented. The answers were associated with a numerical value and the sum of which totaled a profile for both anxiety and depression. For values between zero and 7 the conditions of anxiety and depression were to be considered normal, for values between 8 and 10 the conditions of anxiety and depression were to be considered borderline, for values between 11 and 21 the conditions of anxiety and depression were considered pathological.

The MBI and the HADS validity and psychometric properties were well documented from other previous studies (Maslach et al., 2001; Schaufeli et al., 2001; Kokkinos et al., 2006; Djukanovic et al., 2017). 


\section{Ethical considerations}

The participation to the on-line questionnaire was an implicit consent. All nurses who agreed to participate in the survey returned the questionnaire, while those who disagreed did not return it.

The questionnaire is anonymous to ensure privacy of nurses which is protected.

\section{Methods}

The questionnaire administration

The on-line questionnaire was developed in an anonymous form through the Google function: Google Modules and is administered in the period from 25 Mars 2020 to 25 April 2020 through some pages and nursing groups present on the following Facebook and Instagram pages: \#noisiamopronti, Nurse health professional, Professional nurse, Nurses by passion, NurseTimes, Nurse24.it, Nurse Specialist, Nurseallface, Nursing research, NursesInProgress, Nurses, Active Nurses, Nurses Italy, Nurses supporting health, Nursing Mobility, Nursing Competitions, Informed Nurses (Instagram).

The questionnaire was addressed to all nurses who were engaged in the care of patients with Covid-19, regardless of the assignment department. Nurses who declared that they were at home during this period on different types of leave were excluded.

\section{Data assessment}

Data were collected in an Excel datasheet and statistical analyses were performed using IBM SPSS 20 software. Data were presented as numbers and percentages for all variables considered and differences in the group composition according to variables were evaluated.

Both, for the assessment of burnout and for the state of anxiety and depression, the number of subjects belonging to different risk categories were assessed and groups were created and associated their relative percentages. A multiple comparison chi-square test was used to define significant differences among percentages.

Spearman correlations were assessed to identify significant association among all variables considered in this research.

All tests with $\mathrm{p}<0.05$ were considered significant. 


\section{Results}

In total 400 nurses voluntarily participated in the study (Table 1). Of these $296(74 \%)$ were female and $104(26 \%)$ male. $263(65.75 \%)$ had a working experience that does not exceed 10 years, $71(17.75 \%)$ worked from 11 to 25 years and $66(16.5 \%)$ nurses had a work experience of over 25 years.

Table 1. Sampling characteristics $(n=400)$

\begin{tabular}{rcc}
\hline Characteristics & n (\%) & p value \\
\hline Sex & & \\
Female & $296(74 \%)$ & $.018^{*}$ \\
Male & $104(26 \%)$ & \\
\hline Years of work & & \\
$0-10$ years & $263(65.8 \%)$ & .992 \\
$11-25$ years & $71(17.8 \%)$ & \\
$>26$ years & $66(16.5 \%)$ & \\
\hline
\end{tabular}

Note: $* \mathrm{p}<0.05$. D: Depersonalization; EE: Emotional Exhaustion; HADS: Hospital Anxiety and Depression Scale; MBI: Maslach Burnout Inventory; PA: Personal Accomplishment.

According to sex (Table 2), there were no statistically significant differences in the evaluation of burnout and, specifically, in the evaluation of $\mathrm{EE}(\mathrm{p}=.276)$, in D assessment ( $\mathrm{p}=.732)$, in PA evaluation $(\mathrm{p}=.670)$. In fact, no respondent presented a low risk, $33 \%$ had medium risk (23.75\% females; $9.75 \%$ males) and $67 \%$ had high risk $(50.75 \%$ females; $16.25 \%$ males). For the sub-dimension of D, $77 \%$ had a low risk (57.25\% females; $19.75 \%$ males), $14.3 \%$ a medium risk ( $10 \%$ females; $4.25 \%$ males) and $8.8 \%$ had a high risk $(6.75 \%$ females; $2 \%$ males). Finally, for the subdimension of the PA, $15 \%$ had a low risk (11.5\% females; $3.5 \%$ males), $28.5 \%$ had a medium risk (20.25\% females; $8.25 \%$ males) and $56.5 \%$ had a high risk $(42.25 \%$ females; $14.25 \%$ males).

Also, in the evaluation of anxiety and depression there were no significant differences according to $\operatorname{sex}(p=.257 ; p=.306$, respectively). Specifically, as regards the assessments of the HADS scale, for the state of anxiety $45 \%$ of nurses registered a normal condition (33.75\% females; $11.25 \%$ males), $27.3 \%$ had a borderline state (21.25\% females; $6 \%$ males), $27.8 \%$ had a pathological condition (19\% females; $8.75 \%$ males). As for the data relating to the depressive state, $72.3 \%$ presented as normal (54\% females; $18.25 \%$ males), $16.8 \%$ recorded as borderline $(11.25 \%$ females; $5.5 \%$ males) and $11 \%$ as abnormal condition $(8.75 \%$ females; $2.25 \%$ males). 
Table 2. Burnout, Anxiety and Depression levels according to sex.

\begin{tabular}{rcccc}
\hline Variables & $\begin{array}{c}\text { Total } \\
\mathbf{n}(\%)\end{array}$ & $\begin{array}{c}\text { Female nurses } \\
\mathbf{n}(\%)\end{array}$ & $\begin{array}{c}\text { Male nurses } \\
\mathbf{n}(\mathbf{\%})\end{array}$ & p value \\
\hline MBI-EE: & & & & \\
Low risk & $0(0 \%)$ & $0(0 \%)$ & $0(0 \%)$ & .276 \\
Medium risk & $132(33 \%)$ & $93(23.25 \%)$ & $39(9.75 \%)$ & \\
High risk & $268(67 \%)$ & $203(50.75 \%)$ & $65(16.25 \%)$ & \\
MBI-D: & & & & \\
Low risk & $308(77 \%)$ & $229(57.25 \%)$ & $79(19.75 \%)$ & .732 \\
Medium risk & $57(14.25 \%)$ & $40(10 \%)$ & $17(4.25 \%)$ & \\
High risk & $35(8.75 \%)$ & $27(6.75 \%)$ & $8(2 \%)$ & .670 \\
MBI-PA: & & & & \\
Low risk & $60(15 \%)$ & $46(11.5 \%)$ & $14(3.5 \%)$ & .257 \\
Medium risk & $114(28.5 \%)$ & $81(20.25 \%)$ & $33(8.25 \%)$ & \\
High risk & $226(56.5 \%)$ & $169(42.25 \%)$ & $57(14.25 \%)$ & \\
Normal & $180(45 \%)$ & $135(33.75 \%)$ & $45(11.25 \%)$ & $24(6 \%)$ \\
Borderline & $109(27.25 \%)$ & $85(21.25 \%)$ & $35(8.75 \%)$ & \\
Abnormal & $111(27.75 \%)$ & $76(19 \%)$ & & \\
HADS - Anxiety: & & & \\
Normal & $289(72.25 \%)$ & $216(54 \%)$ & $73(18.25 \%)$ & \\
Borderline & $67(16.75 \%)$ & $45(11.25 \%)$ & $22(5.5 \%)$ & $9(2.25 \%)$ \\
Abnormal & $44(11 \%)$ & $35(8.75 \%)$ &
\end{tabular}

Note: $* \mathrm{p}<0.05$. D: Depersonalization; EE: Emotional Exhaustion; HADS: Hospital Anxiety and Depression Scale; MBI: Maslach Burnout Inventory; PA: Personal Accomplishment.

The same trend could be seen considering the years of work experience (Table 3). As regards the Burnout assessment, specifically for the EE sub-dimension $(\mathrm{p}=.790)$, for $\mathrm{D}(\mathrm{p}=.481)$ and for PA $(\mathrm{p}=.350)$.

In fact, no respondent presented a low risk, $33 \%$ had medium risk $(22.25 \%$ for $0-10$ years of work experience; $5.25 \%$ for $11-25$ years of work experience; $5.5 \%$ for over 26 years of work experience) and $67 \%$ had high risk (43.5\% for $0-10$ years of work experience; $12.5 \%$ for $11-25$ years of work experience; $11 \%$ for over 26 years of work experience). For the sub-dimension of D, 77\% had a low risk $(50.25 \%$ for $0-10$ years of work experience; $13 \%$ for $11-25$ years of work experience; $13.75 \%$ for over 26 years of work experience), $14.3 \%$ a medium risk $(9.75 \%$ for $0-10$ years of work experience; $2.5 \%$ for $11-25$ years of work experience; $2 \%$ for over 26 years of work experience) and $8.8 \%$ had a high risk $(5.75 \%$ for $0-10$ years of work experience; $2.25 \%$ for $11-25$ years of work experience; $0.75 \%$ for over 26 years of work experience). Finally, for the sub-dimension of the PA, $15 \%$ had a low risk (9.5\% for $0-10$ years of work experience; $2 \%$ for $11-25$ years of work experience; $3.5 \%$ for over 26 years of work experience), $28.5 \%$ had a medium risk ( $17.5 \%$ for 0 10 years of work experience; $6 \%$ for $11-25$ years of work experience; $5 \%$ for over 26 years of work experience) and $56.5 \%$ had a high risk (38.75\% for $0-10$ years of 
work experience; $9.75 \%$ for $11-25$ years of work experience; $8 \%$ for over 26 years of work experience).

Also, considering the state of anxiety and depression there were no significant differences between the respondents $(\mathrm{p}=.212 ; \mathrm{p}=.532$, respectively). Specifically, as regards the assessments of the HADS scale, for the state of anxiety $45 \%$ of nurses registered a normal condition $(29.5 \%$ for $0-10$ years of work experience; $9 \%$ for $11-25$ years of work experience; $6.5 \%$ for over 26 years of work experience), $27.25 \%$ had a borderline state $(19.25 \%$ for $0-10$ years of work experience; $3 \%$ for $11-25$ years of work experience; $5 \%$ for over 26 years of work experience), $27.75 \%$ had a pathological condition $(17 \%$ for $0-10$ years of work experience; $5.75 \%$ for $11-25$ years of work experience; $5 \%$ for over 26 years of work experience). As for the data relating to the depressive state, $72.25 \%$ presented as normal (48\% for 0-10 years of work experience; $13 \%$ for $11-25$ years of work experience; $11.25 \%$ for over 26 years of work experience), $16.75 \%$ recorded as borderline $(9.75 \%$ for $0-10$ years of work experience; $3.25 \%$ for $11-25$ years of work experience; $3.75 \%$ for over 26 years of work experience) and $11 \%$ as abnormal condition ( $8 \%$ for $0-10$ years of work experience; $1.5 \%$ for $11-25$ years of work experience; $1.5 \%$ for over 26 years of work experience).

Table 3. Burnout, Anxiety and Depression levels according to years of work experience

\begin{tabular}{|c|c|c|c|c|c|}
\hline Variables & $\begin{array}{l}\text { Total } \\
\mathbf{n}(\%) \\
\end{array}$ & $\begin{array}{c}0-10 \text { years } \\
n(\%)\end{array}$ & $\begin{array}{c}11-25 \text { years } \\
\text { n(\%) }\end{array}$ & $\begin{array}{c}>26 \text { years } \\
\text { n(\%) }\end{array}$ & $p$ value \\
\hline \multicolumn{6}{|l|}{ MBI-EE: } \\
\hline Low risk & $0(0 \%)$ & $0(0 \%)$ & $0(0 \%)$ & $0(0 \%)$ & .790 \\
\hline Medium risk & $132(33 \%)$ & $89(22.25 \%)$ & $21(5.25 \%)$ & $22(5.5 \%)$ & \\
\hline High risk & $268(67 \%)$ & $174(43.5 \%)$ & $50(12.5 \%)$ & $44(11 \%)$ & \\
\hline \multicolumn{6}{|l|}{ MBI-D: } \\
\hline Low risk & $308(77 \%)$ & $201(50.25 \%)$ & $52(13 \%)$ & $55(13.75 \%)$ & .481 \\
\hline Medium risk & $57(14.25 \%)$ & $39(9.75 \%)$ & $10(2.5 \%)$ & $8(2 \%)$ & \\
\hline High risk & $35(8.75 \%)$ & $23(5.75 \%)$ & $9(2.25 \%)$ & $3(0.75 \%)$ & \\
\hline \multicolumn{6}{|l|}{ MBI-PA: } \\
\hline Low risk & $60(15 \%)$ & $38(9.5 \%)$ & $8(2 \%)$ & $14(3.5 \%)$ & .350 \\
\hline Medium risk & $114(28.5 \%)$ & $70(17.5 \%)$ & $24(6 \%)$ & $20(5 \%)$ & \\
\hline High risk & $226(56.5 \%)$ & $155(38.75 \%)$ & $39(9.75 \%)$ & $32(8 \%)$ & \\
\hline \multicolumn{6}{|l|}{ HADS - } \\
\hline Anxiety: & $180(45 \%)$ & $118(29.5 \%)$ & $36(9 \%)$ & $26(6.5 \%)$ & .212 \\
\hline Normal & $109(27.25 \%)$ & $77(19.25 \%)$ & $12(3 \%)$ & $20(5 \%)$ & \\
\hline Borderline & $111(27.75 \%)$ & $68(17 \%)$ & $23(5.75 \%)$ & $20(5 \%)$ & \\
\hline \multicolumn{6}{|l|}{ Abnormal } \\
\hline \multirow{2}{*}{\multicolumn{6}{|c|}{$\begin{array}{r}\text { HADS - } \\
\text { Depression: }\end{array}$}} \\
\hline & & & & & \\
\hline Normal & $289(72.25 \%)$ & $192(48 \%)$ & $52(13 \%)$ & $45(11.25 \%)$ & .532 \\
\hline Borderline & $67(16.75 \%)$ & $39(9.75 \%)$ & $13(3.25 \%)$ & $15(3.75 \%)$ & \\
\hline Abnormal & $44(11 \%)$ & $32(8 \%)$ & $6(1.5 \%)$ & $6(1.5 \%)$ & \\
\hline
\end{tabular}

Note: ${ }^{*} \mathrm{p}<0.05$. D: Depersonalization; EE: Emotional Exhaustion; HADS: Hospital Anxiety and Depression Scale; MBI: Maslach Burnout Inventory; PA: Personal Accomplishment. 
The correlations assessed between all the variables considered in the study highlighted two particular significant positive correlations existing between the state of depression and anxiety $(\mathrm{p}<.001)$ and the sub-dimension of EE with PA $(\mathrm{p}=.039)$. Other significant associations both with socio-demographic variables (sex and years of work experience) and between the dimensions of MBI and HADS were not recorded (Table 4).

Table 4. Spearman correlations between sex, years of work experience, Anxiety, Depression, and MBI sub-dimensions

\begin{tabular}{rccccccc}
\hline & Sex & $\begin{array}{c}\text { Years of } \\
\text { w. e. }\end{array}$ & MBI-E.E. & MBI-D. & MBI-P.A. & $\begin{array}{c}\text { HADS- } \\
\text { Anxiety }\end{array}$ & $\begin{array}{c}\text { HADS- } \\
\text { Depression }\end{array}$ \\
\hline Sex & & & & & & & \\
$\mathrm{R}$ & 1.00 & -.007 & -.057 & .010 & -.009 & .050 & .015 \\
$\mathrm{p}$ & --- & .893 & .258 & .844 & .860 & .323 & .758 \\
\hline $\begin{array}{r}\text { Years of } \\
\text { w. e. }\end{array}$ & & & & & & & \\
$\mathrm{R}$ & -.007 & 1.00 & .019 & -.032 & -.072 & .032 & .017 \\
$\mathrm{p}$ & .893 & --- & .699 & .517 & .148 & .527 & .741 \\
\hline MBI-E.E. & & & & & & & \\
$\mathrm{R}$ & -.057 & .019 & 1.00 & .014 & $.013^{*}$ & .038 & -.009 \\
$\mathrm{p}$ & .258 & .699 & --- & .778 & $.039^{*}$ & .449 & .852 \\
\hline MBI-D. & & & & & & & \\
$\mathrm{R}$ & .010 & -.032 & .014 & 1.00 & .076 & .020 & .021 \\
$\mathrm{p}$ & .844 & .517 & .778 & --- & .129 & .685 & .809 \\
\hline MBI-P.A. & & & & & & & \\
$\mathrm{R}$ & -.009 & -.072 & $.103^{*}$ & .076 & 1.00 & .028 & .075 \\
$\mathrm{p}$ & .860 & .148 & $.039^{*}$ & .129 & --- & .576 & .134 \\
\hline HADS- & & & & & & & \\
Anxiety & & & & & & & \\
$\mathrm{R}$ & .050 & .032 & .038 & .020 & .028 & 1.00 & $.555^{*}$ \\
$\mathrm{P}$ & .323 & .527 & .449 & .685 & .576 & --- & $<.001^{*}$ \\
\hline HADS- & & & & & & & \\
Depression & & & & & & \\
$\mathrm{R}$ & .015 & .017 & -.009 & .012 & .075 & $.555^{*}$ & 1.00 \\
$\mathrm{p}$ & .758 & .741 & .852 & .809 & .134 & $<.001^{*}$ & -- \\
\hline
\end{tabular}

Note: ${ }^{*} \mathrm{p}<0.05$; R: Spearman Correlation Coefficient. D: Depersonalization; EE: Emotional Exhaustion; HADS: Hospital Anxiety and Depression Scale; MBI: Maslach Burnout Inventory; PA: Personal Accomplishment.

\section{Discussion}

The present study aimed to investigate the psychological state of nurses directly involved in the care of patients with Covid-19, regarding the burnout, anxiety and depression levels according to sex and years of work experience. 
Previous studied had showed the same subject, many of which took place in China, as the Covid-19 pandemic originated there (Zhang et al., 2020; Wang et al., 2020; Usher et al, 2020; Vitale et al. 2020).

In the study of Liu et al. (2020) it was concluded that the incidence of nurses' burnout was high in the period of Covid-19, which was closely related to anxiety and depression. When nurses were more anxious and had shorter working years, their burnout was more likely to be stronger. Therefore, both managers and nurses themselves should pay attention to burnout. Nurses with symptoms of anxiety and shorter working time are the focus of attention and intervention.

Although this study analyzes the same variables considered in our work, our data show instead a strong correlation between the levels of anxiety and depression $(\mathrm{p}<0.001)$ and not between the levels of anxiety and the three sub-dimensions of burnout ( $p=.449 ; p=.685 ; p=.576$, respectively). In the Mo et al. (2020) an on-line questionnaire was administered to assess the work stress among Chinese nurses who are supporting Wuhan in fighting against the Covid-19 infection. It was shown that nurses who fight against the covid-19 were generally under pressure. Moreover, the stress load was high as well as the total anxiety score.

Our results instead reported mostly normal or borderline anxiety levels, as 111 nurses presented abnormal anxiety levels. In the systematic review of Pappa et al. (2020), which considered 13 studies with a combined total of 33.062 participants, anxiety was assessed in 12 studies with a pool prevalence of $23.2 \%$ and depression in 10 studies with a relevance rate of $22.8 \%$. A subgroup analysis revealed gender and occupational differences with female nurses and nurses exhibiting higher rates of effective symptoms compared to male and medical staff.

Our data disagreed data showed in the review considered, as 135 female nurses presented normal anxiety levels and 76 female nurses abnormal anxiety levels and 85 females also borderline anxiety values. The trend was the same for male nurses as well. Therefore, it could be inferred that our data fortunately showed a good trend in anxiety and depression levels among nurses, both female and male, engaged in the care of patients with Covid-19.

Moreover, in the $\mathrm{Hu}$ et al. study (2020) it was examined mental health as: burnout, anxiety, depression and fear and their associations among frontline nurses who were caring for Covid-19 patients in Wuhan, China. The study concluded that the frontline nurses experienced a variety of mental health challenges, especially burnout and fear, which warrant attention and support from policymakers. Moreover, authors showed a moderate level of burnout, while our data recorded a significant increase in the size of emotional exhaustion $(\mathrm{p}<0.001)$, which not differed according to $\operatorname{sex}(\mathrm{p}=.276)$ and the years of work experience $(\mathrm{p}=.790)$. The same trend was not recorded in the sub-dimension of depersonalization and personal accomplishment, since data recorded not significant differences also according to socio-demographic variables considered. 
Certainly the experience of the pandemic was very emotional for the nurses engaged in the front line in the care of patients with Covid-19. However, anxiety and depression levels turned out to be normal, while some nurses experienced them in the dimensions of emotional exhaustion

\section{Conclusion}

From the analysis of our data, compared with those of the current literature, it can be concluded that the Italian nurses interviewed presented normal anxiety and depression levels compared to the data present in the literature as regards the other nurses colleagues interviewed in other parts of the world. The levels of anxiety and depression reported in our data were certainly comforting. A little less encouraged were the values of burnout levels, in particular in the dimensions of emotional exhaustion. Therefore, the practical implication concerned the implementation of support policies for nurses in tackling the problem of burnout more than that of anxiety or depression. Finally, since our sample was small, it will be desirable in the future to study with a larger sample size in order to make them better generalizable since the problem addressed concerns thousands of Italian nurses.

\section{References}

Bohlken, J., Schomig, F., Lemke, M.R., Pumberger, M., \& Riedel-Heller, S.G. (2020). COVID-19 pandemic: stress experience of healthcare workers - a short current review. Psychiatric Praxis, 47(4), 190-197.https://doi.org/ 10.1055/a1159-5551.

Chen, J., Li, J., Cao, B., Wang, F., Luo, L., \& Xu, J. (2020). Mediating effects of self-efficacy, coping, burnout, and social support between job stress and mental health among young Chinese nurses. Journal of Advanced Nursing, 76(1), 163173.https://doi.org/10.1111/jan.14208.

Cheung, T., Fong, T.K.H., \& Bressington, D. (2020). COVID-19 under the SARS Cloud: Mental Health Nursing during the Pandemic in Hong Kong. Journal of Psychiatric and Mental Health Nursing.https://doi.org/10.1111/jpm.12639.

Chung, B.P.M., Wong, T.K.S., Suen, E.S.B., \& Chung, J.W.Y. (2005) SARS: caring for patients in Hong Kong. J Clin Nurs., 14, 510-517. https://doi.org/10.1111/ j.1365-2702.2004.01072.x

Craigie, M., Osseiran-Moisson, R., Hemsworth, D., Aoun, S., Francis, K., Brown, J., \& Rees, C. (2016). The influence of trait-negative affect and compassion satisfaction on compassion fatigue in Australian nurses. Psychological Trauma: Theory, Research, Practice, and Policy, 8(1), 88-97. https://doi.org/ $10.1037 /$ tra00 00050 
Djukanovic, I., Carlsson, J., \& Årestedt, K. (2017). Is the Hospital Anxiety and Depression Scale (HADS) a valid measure in a general population 65-80 years old? A psychometric evaluation study. Health Qual Life Outcomes, 15(1),193. https://doi.org/10.1186/s12955-017-0759-9

Du, J., Dong, L., Wang, T., \& et al. (2020). Psychological symptoms among frontline healthcare workers during COVID-19 outbreak in Wuhan. Gen. Hosp. Psychiatry. https://doi.org/10.1016/j.genhosppsych.2020.03.011

Hu, D., Kong Yue, L., Wengang, H.Q., \& et al. Frontline Nurses' Burnout, Anxiety, Depression, and Fear Statuses and Their Associated Factors During the COVID-19 Outbreak in Wuhan, China: A Big-Scale Cross-Sectional Study. Available at SSRN: https://ssrn.com/abstract=3566144 or http://dx.doi.org/ 10.2139/ssrn.3566144

Huang, C., Wang, Y., Li, X., \& et al. (2020). Clinical features of patients infected with 2019 novel coronavirus in Wuhan, China. Lancet, 395(10223), 497-506. https://doi.org/ 10.1016/S0140-6736(20)30183-5.

Kokkinos, C.M. (2006). Factor structure and psychometric properties of the Maslach Burnout Inventory-Educators Survey among elementary and secondary school teachers in Cyprus. Stress and Health, 22(1), 25-33.

Kreuels, B., Wichmann, D., Emmerich, P., Schmidt-Chanasit, J., de Heer, G., Kluge, S., \& et al. (2014). A case of severe Ebola virus infection complicated by gramnegative septicemia. The New England Journal of Medicine, 371, 2394-401. https://doi.org/10.1056/NEJMoa1411677

Lehmann, M., Bruenahl, C.A., Löwe, B., \& et al. (2015). Ebola and psychological stress of health care professionals. Emerging Infectious Diseases, 21(5), 913914. https://doi.org/10.3201/eid2105.141988

Liu, G., \& Zhang, H. (2020). A study on Burnout in Nurses in the Period of COVID19. Psychology and Behavioral Sciences, 9(3), 31-36. https://doi.org/10. 11648/j.pbs.20200903.12

Liu, X., Kakade, M., Fuller, C.J., \& et al. (2012). Depression after exposure to stressful events: lessons learned from the severe acute respiratory syndrome epidemic. Comprehensive Psychiatry, 53(1), 15-23. https://doi.org/ 10.1016/j. comppsych.2011.02.003.

Maslach, C., Schaufeli, W. B., \& Leiter, M. P. (2001). Job burnout. Annual review of psychology, 52(1), 397-422.

Mathieu, F. (2014). Occupational hazards: Compassion fatigue, vicarious trauma and burnout. The Canadian Nurse, 110(5), 12-13.

Mo, Y., Deng, L., Zhang, L., \& et al. (2020). Work stress among Chinese nurses to support Wuhan in fighting against COVID-19 epidemic. Journal of Nursing Management, 00,1-8. https://doi.org/10.1111/jonm.13014

Mok, E., Chung, B.P., Chung, J.W., Wong, T.K. (2005) An exploratory study of nurses suffering from severe acute respiratory syndrome (SARS). Int. J. Nurs. Pract., 11, 150-160. https://doi.org/10.1111/j.1440-172X.2005.00520.x 
Murthy, S., Gomersall, C.D., \& Fowler, R.A. (2020). Care for critically ill patients with COVID-19. JAMA, 323(15), 1499. https://doi.org/10.1001/jama.2020.3633

Pappa, S., Ntella, V., Giannakas, T., Giannakoulis, V.G., Popoutsi, E., \& Katsaounou, P. (2020). Prevalence of depression, anxiety and insomnia among healthcare workers during the COVID-19 pandemic: A systematic review and meta-analysis. Brain, Behavior, and Immunity, 88,901-907. https://oi.org/ 10.1016/j.bbi.2020.05.026

Schaufeli, W.B., Bakker, A.B., Hoogduin, K., Schaap, C., \& Kladler, A. (2001). The clinical validity of the maslach burnout inventory and the burnout measure. Psychology \& Health, 16(5), 565-582. https://doi.org/10.1080/0887044010 8405527

Suzuki, E., Itomine, I., Kanoya, Y., Katsuki, T., Horii, S., \& Sato, C. (2006) Factors Affecting Rapid Turnover of Novice Nurses in University Hospitals. J. Occup, 48, 49-61. https://doi.org/10.1539/joh.48.49

Tan, B.Y.Q., Chew, N.W.S., Lee, G.K.H., \& et al. (2020). Psychological impact of the COVID-19 pandemic on health care workers in Singapore. Annals of Internal Medicine 173(4), 317-320. https://doi.org/10.7326/M20-1083

Usher, K., Durkin, J., \& Bhullar, N. (2020). The COVID-19 pandemic and mental health impacts. International Journal of Mental Health Nursing, 29(3), 315318. https://doi.org/10.1111/inm.12726

Van Zyl, A.B., \& Noonan, I. (2018). The Trojan War inside nursing: An exploration of compassion, emotional labour, coping and reflection. British Journal of Nursing, 27(20), 1192-1196.

Vitale, E., Mea, R., Di Dio, F., Canonico, A., \& Galatola, V. (2020). Anxiety, Insomnia and Body Mass Index scores in Italian nurses engaged in the care of COVID-19 patients. Endocrine, Metabolic \& Immune Disorders Drug Targets, 20,1. E-pubAhead of Print. https://doi.org/10.2174/1871530320666201016150033

Vitale, E., Cesano, E., \& Germini, F. (2020). Correlations of burnout and healthcare safety perceptions among Italian Nurses. Acta Biomedica, 91(4) [Online ahead of print]. https://doi.org/10.23750/abm.v91i4.9008.

Vitale E, Galatola V, \& Mea R. (2020). Exploring within and between gender differences in burnout levels in Italian nurses engaged in the Covid-19 health emergency: a cohort observational study. Minerva Psichiatrica, 61(4), 162170. https://doi.org/10.23736/S0391-1772.20.02090-7

Vitale, E., Moretti, B., Notarnicola, A., \& Covelli, I. (2020). How the Italian nursing student deal the pandemic Covid-19 condition. Acta Biomedica for Health Professions., 91(12-S):e2020007. https://doi.org/10.23750/abm.v91i12-S.9860. PMID: 33263339.

Wang, C., Pan, R., Wan, X., \& et al. (2020). Immediate psychological responses and associated factors during the initial stage of the 2019 coronavirus disease (COVID-19) epidemic among the general population in China. International 
Journal of Environmental Research and Public Health, 17(5):1729. https://doi.org/10.3390/ijerph17051729

Wu, Y., Wang, J., Luo, C., Hu, S., Lin, X., Anderson, A.E., \& Qian, Y. (2020). A comparison of burnout frequency among oncology physicians and nurses working on the front lines and usual wards during the COVID-19 epidemic in Wuhan, China. Journal of Pain and Symptom Management, 60(1),e60-e65. https://doi.org/10.1016/j.jpainsymman.2020.04.008

Zhang, W., Wang, K., Yin, L., \& et al. (2020). Mental health and psychosocial problems of medical health workers during the COVID-19 epidemic in China. Psychotherapy Psychosomatics, 89(4),1-9. https://doi.org/10.1159/000507639. 